CbNU-Th-94-27

June, 1994

\title{
Non-hermitian techniques of canonical transformations in quantum mechanics.
}

\author{
Haewon Lee円 and W. S. l'Yi \\ Department of Physics \\ Ch'ungbuk National University \\ Ch'ongju, 360-763, Ch'ungbuk, Korea
}

\begin{abstract}
The quantum mechanical version of the four kinds of classical canonical transformations is investigated by using non-hermitian operator techniques. To help understand the usefulness of this appoach the eigenvalue problem of a harmonic oscillator is solved in two different types of canonical transformations. The quantum form of the classical Hamiton-Jacobi theory is also employed to solve time dependent Schrödinger wave equations, showing that when one uses the classical action as a generating function of the quantum canonical transformation of time evolutions of state vectors, the corresponding propagator can easily be obtained.
\end{abstract}

PACS number(s): 03.65.-w, 03.65.Ca, 04.20.Fy, 04.60.Ds

\footnotetext{
${ }^{1}$ hwlee@cbucc.chungbuk.ac.kr

${ }^{2}$ wslyi@cbucc.chungbuk.ac.kr
} 


\section{Introduction}

The idea of canonical transformations is one of the highlights of classical mechanics [1]. It is not only theoretically but also practically important, and provides some clue to the quantization of classical systems. But the interesting point is that even though the canonical transformations and the HamiltonJacobi theory are very helpful for solving classical equations of motions there appeared until now no serious attempts to use these ideas while solving quantum mechanical problems. One of the reasons may be that as long as one restricts himself to the unitary forms of canonical transformations there is no room for nontrivial transformations.

It was Anderson who seriously began to doubt the usual usage of the unitary canonical transformations, and he initiated a non-unitary technique of canonical transformations[2]. His idea is that the commutation relations $\left[q_{r}, p_{s}\right]=i \delta_{r s},\left[q_{r}, q_{s}\right]=\left[p_{r}, p_{s}\right]=0$ are preserved not only under unitary transformations but also following similarity transformations

$$
Q_{r}=C q_{r} C^{-1}, \quad P_{s}=C p_{s} C^{-1}
$$

Observing the fact that any canonical transformation can be decomposed into three basic canonical transformations, he computed the eigenvalue equation of harmonic oscillators and also calculated propagators for some model cases. Even though his idea is quite general it lacks clear classical counterparts.

In this paper we follow the traditional "mixed matrix element technique" of canonical transformations [3]. But the novel difference is that our mixed matrix elements are not unitary, thus allowing us to incorporate Anderson's idea of non-unitary canonical transformations. In fact it is shown that the 
quantum versions of canonical transformations exist and formally are similar to the classical ones. Using the full classical properties of canonical transformations we are able to solve the eigenvalue equation of a harmonic oscillator in a canonically-transformed new space. Even the time-dependent Schrödinger equations of free particles and harmonic oscillators can be solved by using the quantum version of the Hamilton-Jacobi theory.

This paper is organized in the following way. Non-unitary quantum canonical transformations which have classical analogies are introduced in chapter two. In chapter three, the quantum version of a classical canonical transformation is used to solve the energy eigenstates of harmonic oscillators. The time evolutions of Schrödinger wave equations of the free particles and the harmonic ocillators are also solved by using the quantum version of the Hamilton-Jacobi theory. Conclusions and further discussions are given in chapter four.

\section{Quantum canonical transformations.}

In classical mechanics there are four different types of canonical transformations, depending on the forms of generating functions $F_{1}\left(q_{r}, Q_{s}, t\right), F_{2}\left(q_{r}, P_{s}, t\right)$, $F_{3}\left(p_{r}, Q_{s}, t\right)$, and $F_{4}\left(p_{r}, P_{s}, t\right)$. Even though some of them are related there are transformations which cannot be described by any other type of transformation. In this paper the quantum versions of the first and second types of canonical transformations are presented. The other two remaining ones can be inferred from these.

\section{a) Canonical transformations of the first kind.}

Suppose $\left|q^{\prime}\right\rangle=\left|q_{1}^{\prime}, \ldots, q_{f}\right\rangle$ is a simultaneous eigenket of observables $q_{r}$, 
$r=1, \ldots, f$, such that

$$
\begin{aligned}
q_{r}\left|q^{\prime}\right\rangle & =q_{r}^{\prime}\left|q^{\prime}\right\rangle, \\
\left\langle q^{\prime} \mid q^{\prime \prime}\right\rangle & =\frac{1}{\rho\left(q^{\prime}\right)} \delta\left(q^{\prime}-q^{\prime \prime}\right), \\
\int d^{f} q^{\prime}\left|q^{\prime}\right\rangle \rho\left(q^{\prime}\right)\left\langle q^{\prime}\right| & =1 .
\end{aligned}
$$

From now on we use the convention that various eigenvalues of an observable $q_{r}$ are denoted by attaching primes such as $q_{r}^{\prime}, q_{r}^{\prime \prime}$, etc. Schrödinger equations are sometimes readily solvable in different basis kets $\left|Q^{\prime} t\right\rangle=\left|Q_{1}^{\prime}, \ldots, Q_{f}^{\prime}, t\right\rangle$ which are defined with respect to $\left|q^{\prime}\right\rangle$ by

$$
\left\langle q^{\prime} \mid Q^{\prime} t\right\rangle=e^{i F\left(q_{r}^{\prime}, Q_{s}^{\prime}, t\right)},
$$

where $F$ is a function of real numbers $q_{r}^{\prime}$ and $Q_{s}^{\prime}$, and time $t$. Transformations of these kinds were investigated from the early days of quantum mechanics [3]. One should note that for an arbitrary function $F$ the completeness condition of $\left|Q^{\prime} t\right\rangle$ is not guaranteed. Transformations which do not satisfy the completeness condition lose some information. Here we consider only the generating functions which meet the completeness condition. General case will considered in appendix B. Suppose that for some density function $\rho\left(Q^{\prime}, t\right)$ they are complete, that is

$$
\begin{aligned}
\left\langle Q^{\prime} t \mid Q^{\prime \prime} t\right\rangle & =\frac{1}{\rho\left(Q^{\prime}, t\right)} \delta\left(Q^{\prime}-Q^{\prime \prime}\right), \\
\int d^{f} Q^{\prime}\left|Q^{\prime} t\right\rangle \rho\left(Q^{\prime}, t\right)\left\langle Q^{\prime} t\right| & =1 .
\end{aligned}
$$

In this case we are able to define other observables $Q_{r}, r=1, \ldots, f$, such as

$$
Q_{r}\left|Q^{\prime} t\right\rangle=Q_{r}^{\prime}\left|Q^{\prime} t\right\rangle .
$$

To get the quantum analogy of classical canonical transformations let us assume that $F\left(q_{r}, Q_{s}, t\right)$ is a "well-ordered" operator in the sense that it is a 
sum of $q$-functions multiplied by $Q$-functions on the right. In that case we have

$$
\left\langle q^{\prime}\left|F\left(q_{r}, Q_{s}, t\right)\right| Q^{\prime} t\right\rangle=F\left(q_{r}^{\prime}, Q_{s}^{\prime}, t\right)\left\langle q^{\prime} \mid Q^{\prime} t\right\rangle
$$

In this case "the non-hermitian canonical momentum operators" $p_{r}$ and $P_{r}$, which are defined by

$$
\begin{aligned}
\left\langle q^{\prime}\left|p_{r}\right| Q^{\prime} t\right\rangle & =-i \frac{\partial}{\partial q_{r}^{\prime}}\left\langle q^{\prime} \mid Q^{\prime} t\right\rangle \\
\left\langle Q^{\prime} t\left|P_{r}\right| q^{\prime}\right\rangle & =-i \frac{\partial}{\partial Q_{r}^{\prime}}\left\langle Q^{\prime} t \mid q^{\prime}\right\rangle
\end{aligned}
$$

can be recast in the familiar forms of classical mechanics

$$
p_{r}=\frac{\partial F}{\partial q_{r}}, \quad P_{r}=-\frac{\partial F^{\dagger}}{\partial Q_{r}}
$$

Note that even if $q_{r}$ and $Q_{s}$ are observables the corresponding canonical momentum operators may not be hermitian. In fact their hermitiain conjugations are

$$
p_{r}^{\dagger}=\rho(q)^{-1} p_{r} \rho(q), \quad P_{r}^{\dagger}=\rho(Q, t)^{-1} P_{r} \rho(Q, t) .
$$

These non-hermitian properties are essential for our investigation and will be discussed more carefully in the last part of this section.

We now proceed to get the Schrödinger equation of motion in the canonicallytransformed $Q$-space. Let $|t\rangle$ be a Schrödinger ket whose motion is given by $i \frac{d}{d t}|t\rangle=H\left(q_{r}, p_{s}, t\right)|t\rangle$. The $Q$-space wave function $\phi\left(Q_{r}^{\prime}, t\right)=\left\langle Q^{\prime} t \mid t\right\rangle$ in terms of $q$-space wave function $\psi\left(q^{\prime}, t\right)=\left\langle q^{\prime} \mid t\right\rangle$ is given by

$$
\phi\left(Q_{r}^{\prime}, t\right)=\int e^{-i F^{*}\left(q_{r}^{\prime}, Q_{s}^{\prime}, t\right)} \psi\left(q_{r}^{\prime}, t\right) \rho\left(q_{r}^{\prime}\right) d^{f} q^{\prime}
$$

Applying the usual wave equation to $\psi\left(q_{r}^{\prime}, t\right)$ one obtains

$$
i \frac{\partial}{\partial t} \phi\left(Q_{r}^{\prime}, t\right)=K\left(Q_{r}^{\prime},-i \frac{\partial}{\partial Q_{s}^{\prime}}, t\right) \phi\left(Q_{r}^{\prime}, t\right),
$$


where the $Q$-space Hamiltonian $K\left(Q_{r}, P_{s}, t\right)$ is

$$
K\left(Q_{r}, P_{s}, t\right)=H\left(q_{r}, p_{s}, t\right)+\frac{\partial F^{\dagger}}{\partial t} .
$$

After solving (12) the true wave function in $q$-space is constructed by

$$
\psi\left(q_{r}^{\prime}, t\right)=\int e^{i F\left(q_{r}^{\prime}, Q_{s}^{\prime}, t\right)} \phi\left(Q_{r}^{\prime}, t\right) \rho\left(Q_{r}^{\prime}, t\right) d^{f} Q^{\prime} .
$$

At this point we would like to emphasize that to get the completeness condition (B.1), oftentimes one is forced to use "wrong" density functions in the original $q$-space. For example, in a one-dimensional case the proper density function $\rho(x)$ of the cartesian coordinate $x$ is constant. But when one uses a generating function of the form $F(x, Q)=x^{3} Q$, he is obliged to employ the wrong density function $\widetilde{\rho}(x)=2 x^{3}$. Even more, because of (10), the hamiltonian becomes non-hermitian. These two problems cancel each other out to resolve the dilemma. This can be seen in the following way. First rescale the original basis kets such as

$$
\widetilde{\left|q^{\prime}\right\rangle}=\left|q^{\prime}\right\rangle S\left(q^{\prime}\right)^{-1}
$$

where

$$
S(q)=\left(\frac{\widetilde{\rho}(q)}{\rho(q)}\right)^{\frac{1}{2}},
$$

and $\widetilde{\rho}(q)$ is a new density function. These rescaled kets satisfy the following completeness conditions

$$
\begin{aligned}
& \widetilde{\left\langle q^{\prime}\right.} \mid \widetilde{\left.q^{\prime \prime}\right\rangle}=\frac{1}{\widetilde{\rho}\left(q^{\prime}\right)} \delta\left(q^{\prime}-q^{\prime \prime}\right), \\
& \int d^{f} q^{\prime} \widetilde{\left|q^{\prime}\right\rangle} \widetilde{\rho}\left(q^{\prime}\right) \widetilde{\left\langle q^{\prime}\right|}=1 \text {. }
\end{aligned}
$$

The corresponding conjugate momentum operator $\widetilde{p}_{r}$ defined by

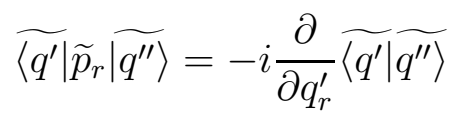


is related to $p_{r}$ by a similarity transformation

$$
\widetilde{p}_{r}=S p_{r} S^{-1}
$$

To investigate how the Schrödinger wave equation changes under this similarity transformation we make use of the standard ket $>$ which is introduced by Dirac in his famous book on quantum mechanics [4]. For a given set of basis kets $\left|q^{\prime}\right\rangle$ it, by definition, satisfies

$$
\left\langle q^{\prime} \mid\right\rangle=1
$$

The reason why one introduces the standard ket is that any state $|1\rangle$ such as $\left\langle q^{\prime} \mid 1\right\rangle=\psi\left(q^{\prime}\right)$ can be written as $\left.\psi(q)\right\rangle$, that is, a function $\psi(q)$ of an observable $q$ operating on the standard ket $\rangle$. When one chooses another set of basis kets $\widetilde{\left|q^{\prime}\right\rangle}$ he may use another standard ket $\widetilde{\rangle}$ such as $\widetilde{\left\langle q^{\prime}\right|} \widetilde{\rangle}=1$. From (15) it is clear that

$$
\tilde{\gamma}=S(q)\rangle
$$

Applying this similarity transformation to the original Schrödinger equation

$$
\left.\left.H\left(q_{r}, p_{s}, t\right) \psi\left(q_{r}, t\right)\right\rangle=i \frac{\partial \psi}{\partial t}\right\rangle
$$

the following similar non-hermitian Schrödinger equation is produced

$$
H\left(q_{r}, \widetilde{p}_{s}, t\right) \psi\left(q_{r}, t\right) \widetilde{\gamma}=i \frac{\partial \psi}{\partial t} \tilde{\gamma} .
$$

It means that as far as one uses a $p_{r}=-i \frac{\partial}{\partial q_{r}}$ representation he may freely choose different density functions while the wave function and the Schrödinger equation remain intact. The sole requirement is that one should use nonhermitian hamiltonian, which is tolerable as far as the wave function is concerned. But the physically meaningful transition probabilities should be constructed by using the original density function at which the hamiltonian is 
hermitian, and so also for the wave function normalization. This fact will be explained when the quantization of the harmonic oscillator is treated.

\section{b) Canonical transformations of the second type.}

Consider two observables $q_{r}$ and $P_{s}$ with corresponding eigenkets $\left|q^{\prime}\right\rangle$ and $\left|P^{\prime} t\right\rangle$ which are related by

$$
\left\langle q^{\prime} \mid P^{\prime} t\right\rangle=e^{i F\left(q_{r}^{\prime}, P_{s}^{\prime}, t\right)}
$$

where the generating function $F\left(q_{r}^{\prime}, P_{s}^{\prime}, t\right)$ is a function of real variables $q_{r}^{\prime}, P_{s}^{\prime}$, and $t$. Since $P_{s}^{\prime}$ is a continuous number, the corresponding ket is normalized by

$$
\left\langle P^{\prime} t \mid P^{\prime \prime} t\right\rangle=\frac{1}{\rho\left(P^{\prime}, t\right)} \delta\left(P^{\prime}-P^{\prime \prime}\right) .
$$

Consider a "well-ordered operator" $F(q, P, t)$ such that

$$
\left\langle q^{\prime}\left|F\left(q_{r}, P_{s}, t\right)\right| P^{\prime} t\right\rangle=F\left(q_{r}^{\prime}, P_{s}^{\prime}, t\right)\left\langle q^{\prime} \mid P^{\prime} t\right\rangle .
$$

The operators defined by

$$
\left\langle q^{\prime}\left|p_{r}\right| P^{\prime} t\right\rangle=-i \frac{\partial}{\partial q_{r}^{\prime}}\left\langle q^{\prime} \mid P^{\prime} t\right\rangle, \quad\left\langle P^{\prime} t\left|Q_{r}\right| q^{\prime}\right\rangle=i \frac{\partial}{\partial P_{r}^{\prime}}\left\langle P^{\prime} t \mid q^{\prime}\right\rangle
$$

can be written as

$$
p_{r}=\frac{\partial F}{\partial q_{r}}, \quad Q_{r}=\frac{\partial F^{\dagger}}{\partial P_{r}} .
$$

The wave equation in $P$-space is

$$
i \frac{\partial}{\partial t} \phi\left(P_{r}^{\prime}, t\right)=K\left(i \frac{\partial}{\partial P_{r}^{\prime}}, P_{s}^{\prime}, t\right) \phi\left(P_{r}^{\prime}, t\right)
$$

where $\phi\left(P_{r}^{\prime}, t\right)=\left\langle P^{\prime} t \mid t\right\rangle$ and $K=H+\frac{\partial F^{\dagger}}{\partial t}$ is a hamiltoinan in $P$-space. 


\section{Applications of quantum canonical transforma- tions.}

As much as the concept of classical canonical transformations is practically helpful for solving equations of motion, so is also the technique of the quantum canonical transformations. It can be used not only for eigenvalue problems, but also for the time evolutions of state vectors including the time-dependent perturbations. In this paper only some ideal physical systems are treated to clarify this rather new concept.

\section{a) Energy eigenfunctions of a harmonic oscillator.}

As an application of type one canonical transformations, consider the following hamiltonian of a harmonic oscillator

$$
H(q, p)=\frac{1}{2}\left(q^{2}+p^{2}\right) .
$$

To solve the energy eigenvalue equation one may choose a generating function

$F(q, Q)=\frac{1}{2} q^{2} \cot Q$ which is well known from classical mechanics. In this case $\left|Q^{\prime}\right\rangle$ is defined by

$$
\left\langle q^{\prime} \mid Q^{\prime}\right\rangle=e^{i \frac{1}{2} q^{\prime 2} \cot Q^{\prime}} .
$$

Since $F$ is an even function of $q$, any wave function in $Q$-space becomes an even function in $q$-space whenever $Q$-space is transformed to $q$-space by using the transition amplitute (31). In fact one may prove that

$$
\int_{0}^{\pi} d Q^{\prime}\left|Q^{\prime}\right\rangle \rho\left(Q^{\prime}\right)\left\langle Q^{\prime}\right|=\mathbf{1}_{+},
$$

where $\rho(Q)=\csc ^{2} Q$, and $\mathbf{1}_{+}$is the parity even projection operator in $Q$-space. One may push along this line only to obtain parity even wave functions. The 
quantization of energy is very intuitive when a generating function of this type is used. (See appendix A for more details.)

To get the complete wave functions the following definition of $Q$ is employed

$$
Q=p+i q
$$

Assuming that it is a type one canonical transformation one can write $p$ as $p=\frac{\partial F(q, Q)}{\partial q}$. Plugging this into (33), $F(q, Q)$ can be solved to give

$$
F(q, Q)=-\frac{i}{2} q^{2}+q Q
$$

Since this generating function contains a bilinear term in $q$ and $Q$, there would be no such problem as the one which we encountered previously. By direct calculations it can be shown that basis kets $\left|Q^{\prime}\right\rangle$ are in fact complete, and the corresponding density functions are

$$
\rho(q)=\frac{1}{2 \pi} e^{-q^{2}}, \quad \rho(Q)=1
$$

The transformed operator $P$ which can be read off from (9) and (34) are

$$
P=-q
$$

The hamiltonian $K$ in $Q$-space is

$$
K=i Q P+\frac{1}{2} Q^{2}+\frac{1}{2}
$$

This hamiltonian is non-hermitian. Even worse, since $p=Q-i q$ is nonhermitian, the original hamiltonian (30) is non-hermitian. All these unsual facts are reflected in the "wrong density functions" given by (35). As we already pointed out in the previous chapter there would be no problem at all 
as far as the wave function is concerned. One may convince himself by checking the final wave function (43).

By (37) the time-independent Schrödinger wave equation in $Q$-space is

$$
\left(Q \frac{\partial}{\partial Q}+\frac{1}{2} Q^{2}+\frac{1}{2}\right) \phi(Q)=E \phi(Q)
$$

This first order differential equation can easily be solved giving the following wave function

$$
\phi(Q)=N Q^{\nu} e^{-\frac{1}{4} Q^{2}},
$$

where $\nu=E-\frac{1}{2}$, and $N$ is a normalization constant which must be determined after the wave function in $q$-space

$$
\psi(q)=N e^{\frac{1}{2} q^{2}} \int_{-\infty}^{\infty} e^{i q Q-\frac{1}{4} Q^{2}} Q^{\nu} d Q
$$

is evaluated. It is not difficult to show that this is proportional to $D_{\nu}(\sqrt{2} q)$, where $D_{\nu}(z)$ is the parabolic cylindrical function defined by [5]

$$
D_{\nu}(z)=\sqrt{\frac{2}{\pi}} 2^{\nu} e^{-\frac{\pi}{2} \nu i} e^{\frac{z^{2}}{4}} \int_{-\infty}^{\infty} x^{\nu} e^{-2 x^{2}+2 i x z} d x, \quad \operatorname{Re} \nu>-1 .
$$

For noninteger $\nu$, it is known that $D_{\nu}(z)$ diverges as $z$ goes to $-\infty[6]$. That means the energy of the hamonic oscillator should be

$$
E=n+\frac{1}{2}, \quad n=0,1, \ldots
$$

The corresponding wave function is

$$
\begin{aligned}
\psi_{n}(q) & =N e^{\frac{1}{2} q^{2}}\left(\frac{1}{i} \frac{d}{d q}\right)^{n} \int_{-\infty}^{\infty} e^{i q Q-\frac{1}{4} Q^{2}} d Q \\
& =N^{\prime}(-)^{n} e^{\frac{1}{2} q^{2}}\left(\frac{d}{d q}\right)^{n} e^{-q^{2}} .
\end{aligned}
$$

This wave function coincides with the usual form constructed directly in the cartesian coordinate system. 


\section{b) Propagators}

Our new technique can also be used to solve the time dependent Schrödinger wave equations. In classical mechanics there is an elegant way of solving equations of motion known as the Hamilton-Jacobi theory, which uses the classical action as the generating function. In that case the transformed hamiltonian vanishes. Since the transformed quantum hamiltonian (13) looks like the classical one, one may expect similar results for quantum mechanics. But because of the noncommutative properties of quantum canonical variables, there may arise terms which are proportional to $\hbar$ which we simply put equal to one.

To convince ourselves the advantage of our quantum Hamilton-Jacobi theory for solving the time-dependent Schrödinger equations, we first consider one-dimensional free particles. The corresponding hamiltonian is

$$
H=\frac{1}{2} p^{2} .
$$

The classical action for $Q$ to $q$ time-evolution during $t$ is

$$
S(q, Q, t)=\frac{(q-Q)^{2}}{2 t} .
$$

That means the best well-ordered quantum generator for this time evolution is

$$
F(q, Q, t)=\frac{1}{2 t}\left(q^{2}-2 q Q+Q^{2}\right) .
$$

Using this generating function the transformed ket $\left|Q^{\prime} t\right\rangle$ is defined by

$$
\left\langle q^{\prime} \mid Q^{\prime} t\right\rangle=e^{i F\left(q^{\prime}, Q^{\prime}, t\right)} .
$$

Note that we are using Schrödinger picture. That is the reason why the original basis ket $\left|q^{\prime}\right\rangle$ is time independent. By the direct calculation we obtain the 
following density functions

$$
\rho(q)=1, \quad \rho(Q, t)=\frac{1}{2 \pi t}
$$

The canonical momentum $p, P$, and the $P$-space hamiltonian $K$ are given by

$$
p=\frac{q-Q}{t}, \quad P=p, \quad K=\frac{i}{2 t} .
$$

Classical hamiltonian in $Q$-space vanishes, but in quantum physics it is propotional to $\hbar$. The equation of motion in $Q$-space is

$$
i \frac{\partial \phi}{\partial t}=\frac{i}{2 t} \phi
$$

and the solution with a convenient normalization constant is

$$
\phi(Q, t)=\sqrt{\frac{2 \pi t}{i}} \phi(Q) .
$$

The true wave equation in $q$-space is therefore

$$
\psi(q, t)=\int_{-\infty}^{\infty} \frac{1}{\sqrt{2 \pi i t}} e^{i \frac{(q-Q)^{2}}{2 t}} \phi(Q) d Q
$$

To understand the physical meaning of this propagation equation we investigate $t \rightarrow 0$ limit. With the help of

$$
\lim _{t \rightarrow 0} \frac{e^{i \frac{\left(x^{\prime}-x^{\prime \prime}\right)^{2}}{2 t}}}{\sqrt{2 \pi i t}}=\delta\left(x^{\prime}-x^{\prime \prime}\right)
$$

it is clear that $\phi(q)$ is nothing but $\psi(q, 0)$. It means that the free particle propagator is, by $(52)$,

$$
G\left(q^{\prime}, t \mid q^{\prime \prime}, 0\right)=\frac{1}{\sqrt{2 \pi i t}} e^{i \frac{\left(q^{\prime}-q^{\prime \prime}\right)^{2}}{2 t}} .
$$

At this point we would like to emphasize that by a direct path integral it is known that the general form of a propagator is [0]

$$
G\left(q^{\prime}, t \mid q^{\prime \prime}, 0\right)=f(t) e^{i S_{c l}}
$$


where $S_{c l}$ is the classical action and $f(t)$ is an undetermined function of time. In our approach $f(t)$ is related to the density function $\rho(Q, t)$ and the $\hbar$ proportional hamiltonian $K$ in $Q$-space.

Using a similar technique one can solve the time-dependent Schrödinger equation for harmonic osillators. The well-ordered generating function would be

$$
F(q, Q, t)=\frac{1}{2}\left(q^{2}+Q^{2}\right) \cot t-q Q \csc t
$$

which is just the classical action for $Q$ to $q$ time-evolution during $t[1]$. One can prove the completeness condition for $\left|Q^{\prime}, t\right\rangle$, obtaining simultaneously the following density functions

$$
\rho(q)=1, \quad \rho(Q, t)=\frac{1}{2 \pi \sin t} .
$$

If $q$ and $Q$ commute, the tranformed hamiltonian $K$ vanishes. But for quantum theory it does not but is proportional to $\hbar$, that is,

$$
K(Q, P, t)=\frac{i}{2} \cot t
$$

Using this hamiltonian the Schrödinger equation in $Q$-space can be solved giving the following time-dependent wave function,

$$
\phi(Q, t)=\sqrt{\frac{2 \pi \sin t}{i}} \phi(Q) .
$$

With the help of (57) and (53) one obtains following propagator of a harmonic oscillator

$$
G\left(q^{\prime}, t \mid q^{\prime \prime}, 0\right)=\frac{1}{\sqrt{2 \pi i \sin t}} e^{i F\left(q^{\prime}, q^{\prime \prime}, t\right)},
$$

where $F\left(q^{\prime}, q^{\prime \prime}, t\right)$ is the classical action given by (56).

To see why the quantum generating function which has the classical analogy is so successful, consider the following classical generating function for 
infinitesmal time evolution

$$
F\left(q_{r}, P_{s}\right)=\sum_{r} q_{r} P_{r}-\delta t H\left(q_{r}, P_{s}\right),
$$

where $H=\frac{1}{2} \sum_{r} P_{r}^{2}+V\left(q_{s}\right)$ is the usual hamiltonian. Using this we define a state $\left|P^{\prime}\right\rangle$ by $\left\langle q^{\prime} \mid P^{\prime}\right\rangle=e^{i F\left(q^{\prime}, P^{\prime}\right)}$. Then the density functions both in $q$ and $P$ spaces are trivial and $Q_{r}$, which is defined by $\frac{\partial F^{\dagger}}{\partial P_{r}}$, is hermitian. It means that all the eigenkets $\left|Q^{\prime}\right\rangle$ form a complete set. To get the physical meaning of $\left|Q^{\prime}\right\rangle$ consider $\left\langle q^{\prime} \mid Q^{\prime}\right\rangle$. Using the completeness of eigenkets $\left|P^{\prime}\right\rangle$ one has

$$
\begin{aligned}
\left\langle q^{\prime} \mid Q^{\prime}\right\rangle & =\int_{-\infty}^{\infty} \frac{d P^{\prime}}{2 \pi}\left\langle q^{\prime} \mid P^{\prime}\right\rangle\left\langle P^{\prime} \mid Q^{\prime}\right\rangle \\
& =\int_{-\infty}^{\infty} \frac{d P^{\prime}}{2 \pi} e^{i \sum q_{r}^{\prime} P_{r}^{\prime}-i \delta t H\left(q_{r}^{\prime}, P_{s}^{\prime}\right)}\left\langle P^{\prime} \mid Q^{\prime}\right\rangle .
\end{aligned}
$$

Now the term $e^{i \sum q_{r}^{\prime} P_{r}^{\prime}}$ in the last part of this equation can be written as $\left\langle Q^{\prime} \leftarrow q^{\prime} \mid P^{\prime}\right\rangle$, where $\left|Q^{\prime} \leftarrow q^{\prime}\right\rangle$ is an eigenket of $Q_{r}$ whose eigenvalue is $q_{r}^{\prime}$. Using this fact (62) can be simplified as

$$
\left\langle q^{\prime} \mid Q^{\prime}\right\rangle=\left\langle Q^{\prime} \leftarrow q^{\prime}\left|e^{-i \delta t H\left(Q_{r}, P_{s}\right)}\right| Q^{\prime}\right\rangle
$$

that is $\left|Q^{\prime} \leftarrow q^{\prime}\right\rangle=e^{-i \delta t H}\left|q^{\prime}\right\rangle$. This mean that (61) is a both classically and quantum mechanically correct generating function of the infinitesmal time evolutions.

\section{Conclusion and discussion}

We would like to emphasize that when the quantum analogies of classical canonical transformations are seriously employed, one obtains useful quantum canonical transformations which can be used to solve either time-independent or time-dependent Schrödinger equations. Furthermore the generating operators of quantum canonical transformations can be inferred from the classical 
generating functions. In this way the classical canonical transformations have some part in quantum mechanics. This classical analogy is our strong point which becomes rather obscure when one uses abstract similarity transformation formalism. (See appendix B for more details.) We expect that our idea will produce more fruitful results when applied to the pertubation theory of quantum mechanics.

\section{Acknowledgments}

This is supported by the Basic Science Research Institute Program of the Ministry of Education, Korea, 1993. 


\section{Appendix}

\section{A. Energy quantizations of harmonic oscillators}

Consider a harmonic oscillator whose hamiltonian is given by

$$
H(q, p)=\frac{1}{2}\left(q^{2}+p^{2}\right)
$$

To get some insight into the energy quantization of a harmonic oscillator we introduce the following transformation

$$
\left\langle q^{\prime} \mid Q^{\prime}\right\rangle=e^{i \frac{1}{2} q^{\prime 2} \cot Q^{\prime}}
$$

Because of (32) this transformation is complete only for the parity even subspace of $\psi(q)$. The corresponding non-hermitian operators $p$ and $P$ are given by

$$
\begin{aligned}
p & =\frac{\partial F}{\partial q}=q \cot Q \\
P & =-\frac{\partial F^{\dagger}}{\partial Q}=\frac{1}{2} \csc ^{2} Q q^{2}
\end{aligned}
$$

From the commutation relation $[q, p]=i$ we have the following equation

$$
[q, \cot Q]=i q^{-1}
$$

Using this relation the $Q$-space hamiltonian $K(Q, P)=H(q, p)$ can be shown to be

$$
K(Q, P)=P+\frac{3}{2} i \cot Q
$$

The eigenvalue equation $K(Q, P) \varphi=E \varphi$ in $Q$-space is therefore

$$
-i\left(\frac{\partial}{\partial Q}-\frac{3}{2} \cot Q\right) \varphi=E \varphi .
$$


It can immediately be solved giving

$$
\varphi(Q)=N \sin ^{\frac{3}{2}} Q e^{i E Q}
$$

where $N$ is a normalization constant. Since $\left|Q^{\prime}\right\rangle$ is defined by (A.2) the wave function in $Q$-space should satisfy $\varphi\left(Q^{\prime}+\pi\right)=\varphi\left(Q^{\prime}\right)$. This means that

$$
e^{i\left(\frac{3}{2}+E\right) \pi}=1
$$

This together with the positive energy condition yields

$$
E=\left(2 n+\frac{1}{2}\right), n=0,1,2, \ldots
$$

The wave function $\psi\left(q^{\prime}\right)$ in $q$ space is given by

$$
\begin{aligned}
\psi_{2 n}\left(q^{\prime}\right) & =N^{\prime} \int_{0}^{\pi} d Q^{\prime} \rho\left(Q^{\prime}\right)\left\langle q^{\prime} \mid Q^{\prime}\right\rangle \varphi\left(Q^{\prime}\right) \\
& =N^{\prime \prime} \int_{0}^{\pi} d Q^{\prime} \frac{1}{\sin ^{\frac{1}{2}} Q^{\prime}} \exp \left(\frac{i}{2} q^{\prime 2} \cot Q^{\prime}+i\left(2 n+\frac{1}{2}\right) Q^{\prime}\right) .
\end{aligned}
$$

When we compare this equation with the well known result for a harmonic oscillator $(60)$

$$
\sum_{n=0}^{\infty} \psi_{2 n}\left(q^{\prime}\right) \psi_{2 n}^{*}(0) e^{-i\left(2 n+\frac{1}{2}\right) T}=\left(\frac{1}{2 \pi i}\right)^{\frac{1}{2}} \frac{e^{\frac{i}{2} q^{\prime 2} \cot T}}{\sin ^{\frac{1}{2}} T},
$$

we obtain the correct parity even wave functions.

\section{B. Generalizations and relation to similarity transformation ap- proach}

In appendix $\mathrm{B}$ we generalize some results obtained in chapter two and investigate the relation between our method and the similarity transformation method given by (11). For simplicity we consider only a one-dimensional case. 
Suppose $\left|q^{\prime}\right\rangle$ be a set of ket vectors in a Hilbert space and $\widetilde{\left|q^{\prime}\right\rangle}$ a new set of ket vectors in the same Hilbert space. In general $\widetilde{\left|q^{\prime}\right\rangle}$ may not form a complete orthogonal set. One can make $\widetilde{\left|q^{\prime}\right\rangle}$ complete by introducing a new inner product in the same Hilbert space, which will be shown later in this appendix. In this case the original $\left|q^{\prime}\right\rangle$ and the new $\widetilde{\left|q^{\prime}\right\rangle}$ will be complete with respect to two different inner products, that is, with respect to the old and to the new ones, respectively. Then the dual vector $\widetilde{\left\langle q^{\prime}\right|}$ should be defined using the new inner product. Suppose that $\left|q^{\prime}\right\rangle$ and $\widetilde{\left|q^{\prime}\right\rangle}$ are complete with density functions $\rho\left(q^{\prime}\right)$ and $\widetilde{\rho}\left(q^{\prime}\right)$, respectively. Then one get

$$
\begin{aligned}
& \int d q^{\prime}\left|q^{\prime}\right\rangle \rho\left(q^{\prime}\right)\left\langle q^{\prime}\right|=1, \\
& \int d q^{\prime} \widetilde{\left|q^{\prime}\right\rangle} \widetilde{\rho}\left(q^{\prime}\right) \widetilde{\left\langle q^{\prime}\right|}=1 \text {. }
\end{aligned}
$$

For some cases $\left|q^{\prime}\right\rangle$ or $\widetilde{\left|q^{\prime}\right\rangle}$ may be incomplete. In that case the left hand side of (团) should be replaced by appropriate projection operators. The operators $q$ and $\widetilde{q}$ which are defined by

$$
q\left|q^{\prime}\right\rangle=q^{\prime}\left|q^{\prime}\right\rangle, \quad \widetilde{q} \widetilde{\left.q^{\prime}\right\rangle}=q^{\prime} \widetilde{\left.q^{\prime}\right\rangle}
$$

will be hermitian with respect to the old inner product and the new one, respectively. It can be shown that the two density functions together with $\widetilde{\left|q^{\prime}\right\rangle}$ determine the new inner product uniquely. To clarify this point, let us consider the followings.

Let us write $\widetilde{\left|q^{\prime}\right\rangle} \equiv C(t)\left|q^{\prime}\right\rangle$, where $C(t)$ can be regarded as a timedependent similarity transformation. Then $\widetilde{q}=C q C^{-1}$. A new dual vector $\widetilde{\left\langle q^{\prime}\right|}$ can be expressed in terms of the old one as

$$
\widetilde{\left\langle q^{\prime}\right|}=\left\langle q^{\prime}\right| C^{\dagger} M
$$


where $M$ is an operator defining the new inner product. In other words, $(\alpha, \beta)=\langle\alpha|M| \beta\rangle$, where $($,$) denotes the new inner product. Using the com-$ pleteness relation we can find

$$
C^{\dagger} M C=\frac{\rho(q)}{\widetilde{\rho}(q)}
$$

and conclude that $\rho, \widetilde{\rho}$ and $C$ determine $M$ completely. In chapter two we specialized ourselves to the cases where both of $\left|q^{\prime}\right\rangle$ and $\widetilde{\left|q^{\prime}\right\rangle}$ form complete orthogonal sets with respect to the same inner product. In this case, $M=1$ and $C$ is "almost unitary" in the sense that $C \sqrt{\frac{\widetilde{\rho}}{\rho}}$ is unitary. This is not always true. As shown above, in general cases we are forced to introduce two different inner products.

Now we study how the momentum operators transform. Using (B.4) and the fact that momentum operators are defined by

$$
\left\langle q^{\prime}\right| p=-i \frac{\partial}{\partial q^{\prime}}\left\langle q^{\prime}\right|, \widetilde{\left\langle q^{\prime}\right| \widetilde{p}}=-i \frac{\partial}{\partial q^{\prime}} \widetilde{\left\langle q^{\prime}\right|},
$$

one can easily prove that

$$
\widetilde{p}=\widetilde{C} p \widetilde{C}^{-1}, \quad \widetilde{q}=\widetilde{C} q \widetilde{C}^{-1}
$$

with $\widetilde{C}=\left(C^{\dagger} M\right)^{-1}$. Next we consider the Schrödinger equation

$$
H(q, p, t)|t\rangle=i \frac{d}{d t}|t\rangle .
$$

Multiplying both sides by $\widetilde{\left\langle q^{\prime}\right|}$, we have

$$
\left.\left.\widetilde{\left\langle q^{\prime}\right.}|H(q, p, t)| t\right\rangle=i \frac{\partial}{\partial t} \widetilde{\left\langle q^{\prime}\right.}|t\rangle-\widetilde{\left\langle q^{\prime}\right.}|\dot{G}| t\right\rangle,
$$

where $\dot{G}$ is defined by

$$
\widetilde{\left\langle q^{\prime}\right|} \dot{G}=i \frac{\partial}{\partial t} \widetilde{\left\langle q^{\prime}\right|}
$$


One can express $\dot{G}$ using the similarity tranformation,

$$
\dot{G}=i \widetilde{C} \frac{\partial}{\partial t} \widetilde{C}^{-1}
$$

Denoting

$$
K(\widetilde{q}, \widetilde{p}, t) \equiv H(q, p, t)+\dot{G},
$$

one can write the Schrödinger equation in $\widetilde{q}$-space as

$$
K\left(q^{\prime},-i \frac{\partial}{\partial q^{\prime}}, t\right) \widetilde{\left\langle q^{\prime} \mid t\right\rangle}=i \frac{\partial}{\partial t} \widetilde{\left\langle q^{\prime} \mid t\right\rangle}
$$

To find $K(\widetilde{q}, \widetilde{p}, t)$ in terms of $\widetilde{q}$ and $\widetilde{p}$, (B.6) may, in principle, be used. However as in chapter two, some interesting results can be obtained if the transformation is expressed in the form

$$
\left\langle q^{\prime} \widetilde{\left.q^{\prime \prime}\right\rangle}=e^{i F\left(q^{\prime}, q^{\prime \prime}, t\right)}, \widetilde{\left\langle q^{\prime \prime}\right.} \mid q^{\prime}\right\rangle=e^{-i G\left(q^{\prime \prime}, q^{\prime}, t\right)}
$$

where $F$ and $G$ are some functions obeying the relation

$$
\int d q^{\prime \prime} e^{i F\left(q^{\prime}, q^{\prime \prime}, t\right)} \widetilde{\rho}\left(q^{\prime \prime}, t\right) e^{-i G\left(q^{\prime \prime}, q^{\prime \prime \prime}, t\right)}=\frac{\delta\left(q^{\prime}-q^{\prime \prime \prime}\right)}{\rho\left(q^{\prime}\right)} .
$$

As in chapter two we assume that $F(q, \widetilde{q}, t)$ and $G(\widetilde{q}, q, t)$ are "well-ordered" operators in the sense that in $F(q, \widetilde{q}, t)$ all $q$ 's are on the left, and in $G(\widetilde{q}, q, t)$ all q's are on the right. Then (9) corresponds to

$$
p=\frac{\partial}{\partial q} F(q, \widetilde{q}, t), \quad \widetilde{p}=-\frac{\partial}{\partial \widetilde{q}} G(\widetilde{q}, q, t),
$$

and we also have

$$
\dot{G}=\frac{\partial}{\partial t} G(\widetilde{q}, q, t)
$$

Eq.(B.15) can be used to get $\widetilde{q}$ and $\tilde{p}$ in terms of $q$ and $p$. In "almost unitary" cases considered in chapter two, we get

$$
G(\widetilde{q}, q, t)=F(q, \widetilde{q}, t)^{\dagger}
$$

The two density functions can be absorbed into $F$ and $G$, which is obvious from (B.14-16). In this case we have $\rho=1$ and $\widetilde{\rho}=1$. 


\section{References}

[1] H. Goldstein, Classical Mechanics, 2nd. ed. (Addsison-Wesley, Massaschusetts, 1980) ; V. I. Arnold, Mathematical Methods of Classical Mechanics (Springer-Verlag, New York, 1978) ; E. J. Saletan and A. H. Cromer, Theoretical Mechanics (John Wiley \& Sons, Inc, New York, 1971) ; E. C. G. Sudarshan and N. Mukunda, Classical Dynamics: A Modern perspective (John Wiley \& Sons, Inc, New York, 1974) ; F. Scheck, Mechanics (Springer-Verlag, Berlin, 1990) ; R. Abraham and J. E. Marsden, Foundations of Mechanics, 2nd. ed. (Addison-Wesley Pub. Co., Inc., Redwood City, California, 1990) .

[2] A Anderson, McGill 92-29, Imperial-TP-92-93-21, NSF-ITP-93-61, hepth/9305054; Phys. Lett. B 305, 67 (1993); 319, 157 (1993); Phys. Rev. A 43, 4602 (1991).

[3] P. A. M. Dirac, Phys. Zeit. der Sowjetunion, Band 3, Heft 1 (1993); J. Schwinger, Phys. Rev. 82 (1951) 914; 91 (1953) 713; P. Ramond, Field Theory: A Modern Primer, 2nd. ed. ( Addison-Wesley Pub. Co., Inc., Redwood City, California ,1990) .

[4] P. A. M. Dirac, The Principles of Quantum Mechanics, 4th. ed. (Oxford University Press, London, 1958) .

[5] I. S. Gradshteyn and I. M. Ryzhik, Table of Integrals, Series, and Products, 4th. ed. (Academic Press, New York, 1980).

[6] P. M. Morse and H. Feshbach Methods of Theoretical Physics (McGrawHill Book Co., New York, 1953) ; E. Merzbacher, Quantum Mechanics, 2nd. ed. (John Wiley \& Sons, New York, 1970) . 
[7] R. P. Feynmann and A. R. Hibbs, Quantum Mechanics and Path Integrals (McGraw-Hill Book Co., New York, 1965) 